\title{
Total Hip Arthroplasty in an Inveterate Femoral Neck Fracture in a Patient with Congenital Insensitivity to Pain with Anhidrosis
}

\author{
Augusto Dagnino $^{1}$ Nicola Ursino ${ }^{2}$ Carlo A. M. Ripamonti ${ }^{2}$ Carlo E. Fiorentini ${ }^{2}$ Michele Scelsi ${ }^{2}$ \\ Riccardo D’Ambrosi ${ }^{2,3}$ Nicola M. Portinaro ${ }^{3,4}$
}

1 Istituto Clinico San Siro, Milan, Italy

2I.R.C.C.S. Istituto Ortopedico Galeazzi, U.O. CASCO, Milan, Italy

3 Università degli Studi di Milano, Milan, Italy

${ }^{4}$ Istituto Clinico Humanitas, U.O. Ortopedia Pediatrica, Milan, Italy

Address for correspondence Augusto Dagnino, MD, Istituto Clinico San Siro, Milan, Italy (e-mail: dr.dagnino@gmail.com).

Joints 2017;5:249-252.

\begin{abstract}
Keywords

- congenital insensitivity to pain with anhidrosis

- total hip arthroplasty

- femoral neck fracture

- dual mobility cup

- hereditary sensory autonomic neuropathy Congenital insensitivity to pain with anhidrosis (CIPA) is an extremely rare disorder characterized by autonomic and sensory nerves malfunction with insensitivity to both deep and superficial painful stimuli, inability to sweat and produce tears, and mild to moderate mental retardation with self-mutilating behavior. Related consequences of inveterate musculoskeletal injuries represent a major issue for these patients, since pain cannot act as a protection mechanism. For the same reason, the patients are at risk during postoperative rehabilitation, which should be taken into account when selecting an orthopaedic implant. To our knowledge, only one case of total hip arthroplasty has been reported in the literature to date. A 21-year-old Caucasian male patient affected with CIPA arrived at our attention complaining about a functional limitation of the left hip. No history of trauma was reported. The X-rays showed an inveterate femoral neck fracture with a severe necrosis and resorption of the femoral head. We decided to perform a total hip arthroplasty with a cemented stem and a cemented dual mobility cup. The postoperative course and rehabilitation were satisfactory, with excellent clinical results, measured with the Harris Hip Score at 1 year.
\end{abstract}

\section{Introduction}

From a biological point of view, pain is a necessary protection mechanism for our tissues, and a lack of pain perception may cause severe problems. There are two groups of conditions that are characterized by the congenital absence of pain perception: hereditary sensory autonomic neuropathy (HSAN) and hereditary nonneuropathic analgesia (HNNA). ${ }^{1} \mathrm{HSAN}$ is a clinically and genetically heterogeneous group of disorders of the peripheral nervous system characterized by congenital malfunction of sensory and autonomic nerves. In 1993, Dyck ${ }^{2}$ classified HSANs into five types by taking into account their functional aspect and other factors, such as mental retardation (when tested, intelligence quotients [IQs] varied from 41 to 78 , the majority being in the 60s; microcephaly was sometimes reported), oral lesions, touch/temperature sensibility, tendon reflexes, production of tears/perspiration, and histopathology of the nerves. On the other hand, patients affected by HNNA have an adequate transmission of the painful stimuli, although they are unable to elaborate them centrally. They are usually normal with respect to intelligence, motor development, nerve histology, and perception of other senses compared with their siblings., 3

Congenital insensitivity to pain with anhidrosis (CIPA), also known as HSAN type 4, is an extremely rare autosomal recessive disorder caused by a polymorphic mutation of the gene, NTRK1 that encodes a receptor for the nerve growth factor (NGF). The mutation leads to autonomic and sensory nerves malfunction with insensitivity to both deep and superficial painful stimuli, inability to sweat and produce tears, and mild to moderate mental retardation with selfmutilating behavior. It was initially described as a syndrome where the entire body was affected by congenital absence of pain sensitivity, although other sensory modalities were intact or minimally impaired and tendon reflexes were published online September 12, 2017
DOI https://doi.org/ 10.1055/s-0037-1606619. ISSN 2282-4324.
Copyright (c) 2017 Georg Thieme Verlag License terms KG Stuttgart · New York 
present. ${ }^{5}$ Children affected by CIPA fail to sweat (anhidrosis) and produce tears and suffer from multiple fever episodes, corneal ulceration with poor healing potential, and repeated traumatic and thermal injuries. Height and weight are below normal percentile for their age. The oral manifestations include premature loss of teeth due to biting hard toys, lips, and fingers. ${ }^{3,6-8}$ Intraoral fibrous scars in cheeks decrease the mouth opening. Abnormalities of the joints (Charcot joints) are often present and must be surgically corrected. Hyperthermia is a major cause of death during the initial years. As these children grow, accidental injuries, such as falls or burns lead to multiple scars, as well as articular dislocations and bone joint fractures that can ultimately predispose to deep infections. ${ }^{8}$ Most of the complications occur at the lower limbs, although all joints can be affected. Fractures occur in the early years of life, especially between the age of 4 and 6 years, due to intense activity. The causes of most fractures and dislocations are not well documented; some are caused by minor trauma, such as short falls. This suggests that CIPA patients may sustain injuries from minor traumas that would not result in injuries in normal children. Video gait analysis of young patients affected by CIPA reported an altered walking, which may contribute to the high incidence of injuries in the lower limbs. ${ }^{9}$

This report presents the surgical treatment for a severe osteonecrosis of the femoral head, secondary to an inveterate neck fracture, in a young male affected by CIPA.

\section{Case Presentation}

The particular form of this case report and the fact that no experimentation was performed made the approval of the local ethics committee unnecessary. Nonetheless, all the authors declared to have managed this case in respect of the Italian laws and of the Declaration of Helsinki.

Our patient, a 21-year-old Caucasian boy, was the first child of consanguineous parents (second-degree cousins). He was delivered normally after an uneventful pregnancy, weight at birth was $3.250 \mathrm{~kg}$. From the first week of life, he had several unexplained bouts of fever, and as reported by his mother, he was always "hot and dry." At 10 months of age, when he began to crawl, the parents noticed bruises with some ulceration on him. At that age, he was diagnosed with CIPA and from that moment on, very accurate care was provided to him by his parents, relatives, and all his teachers with high attention on trauma during the school and game. Luckily, he was able to grow until the age of 18 years without having major injury, except for some dental problems and an episode of traumatic corneal lesion at the age of 11 years.

He arrived at our attention for a functional limitation of the left hip, referring that he was unable to walk for more than two or three steps. At admission in our orthopaedic department, he appeared as an aware and cooperative guy with dry warm skin. His body temperature was $36.7^{\circ} \mathrm{C}$, height was $166 \mathrm{~cm}$, weight was $63 \mathrm{~kg}$, and head circumference was within normal limits. The teeth were almost all present with slightly hypertrophic gums. There were some very small ulcerations in the internal side of the cheeks. Evaluation of intellectual development was a bit difficult because of his shyness. Tested IQ was 76 . Neurologic examination founded moderately depressed tendon reflexes and a lack of reaction to painful stimuli, such as pinpricks or compression of the muscles or tendons. However, he seemed to perceive tactile stimuli. Sensitivity to temperature or vibration was not tested. Routine blood count, serum electrolyte, coagulation, and hepatic and renal function values were normal. At clinical examination, the left hip was slightly shortened $(0.5 \mathrm{~cm})$, abducted, and externally rotated with a limited range of motion (ROM).

Chest X-Ray and ECG did not show alterations. X-rays of the pelvis and left hip showed a severe necrosis of the femoral head with deformation and resorption in an inveterate femoral neck fracture (-Fig. 1). Harris Hip Score (HHS) was 61 points.

Despite the young age of the patient, we decided to perform a total hip arthroplasty (THA) through a posterolateral approach. A severe osteoporosis was found intraoperatively with a total resorption of the femoral head ( - Fig. 2); therefore, we opted for a cemented stem (MS-30 size 6 with $22 \mathrm{~mm} /+0$ metal head; Zimmer Biomet Inc; Warsaw, Indiana, United States) and a cemented double mobility cup (UHL diameter 44/22 mm; Groupe Lépine; Lyon, France), both fixed with antibiotic-loaded cement (GMV gentamicin cement; Johnson
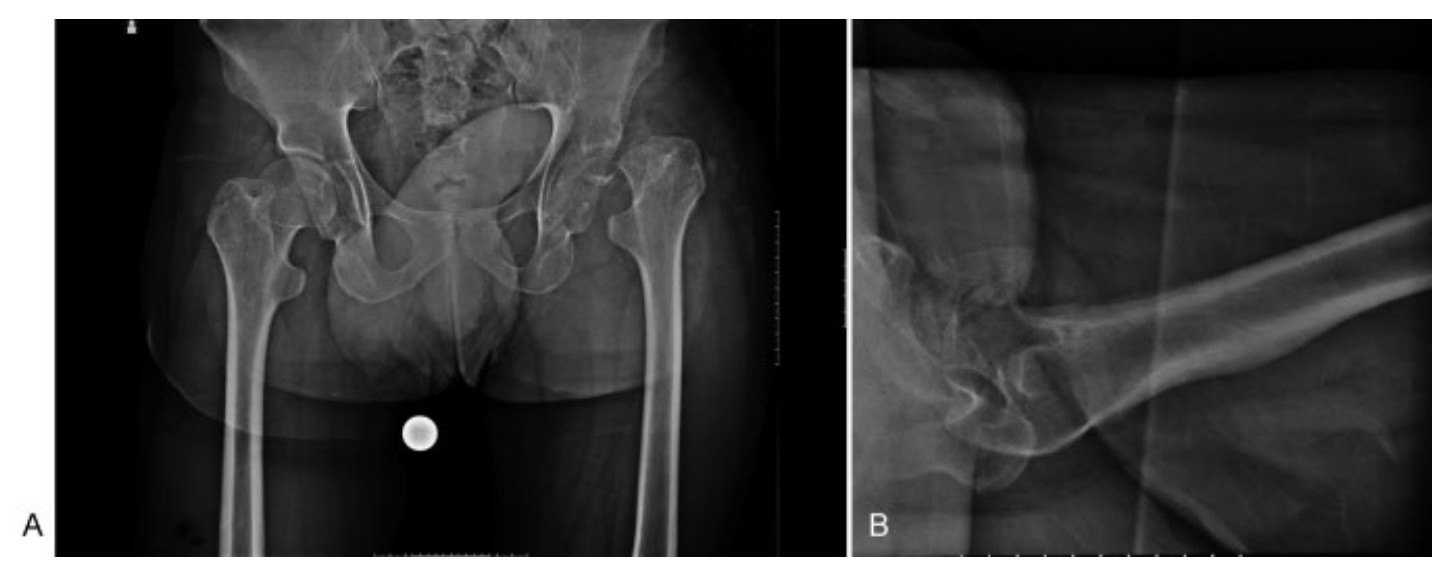

Fig. 1 Anteroposterior weight-bearing radiograph of the pelvis (A) and axial radiograph of the left hip (B) showing a severe necrosis of the left femoral head with deformation and resorption in an inveterate femoral neck fracture. 


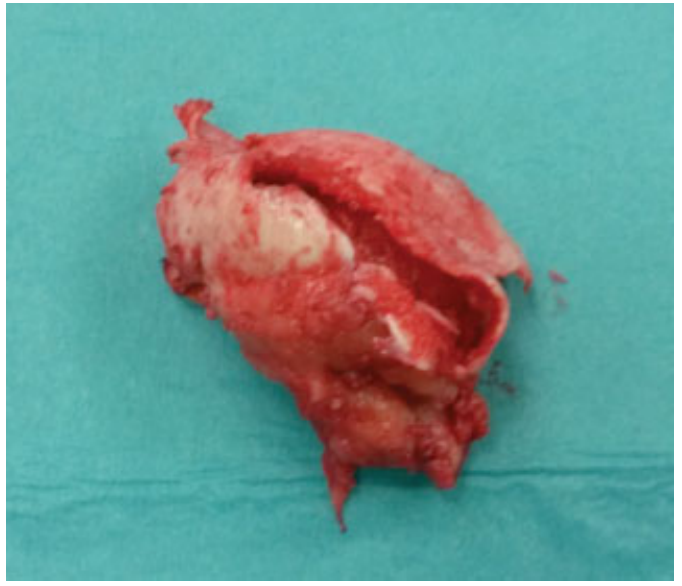

Fig. 2 Intraoperative image showing a severe osteoporosis with a total resorption of the femoral head.

\& Johnson Depuy; Warsaw, Indiana, United States; - Fig. 3). Cemented implant provided an immediate weight bearing to the patient, unabled otherwise to handle partial loads, and reduced the risk of aseptic loosening that would not be referred by the patient.

The postoperative course was uneventful, without thermal rises and with good recovery of the ROM. Antiembolism prophylaxis was administered with 4,000 I.U. of low-molecular-weight heparin for 40 days following our standard protocol. Patients with CIPA reported a higher risk of infection due to a radical shift in body temperature, mainly dictated by environmental temperature, a feature that is only partially explained by the inability to sweat due to defective sweat gland innervation. For this reason, ${ }^{10}$ we opted for a prolonged double antibiotic prophylaxis with a third generation cephalosporin and a fluoroquinolone for 6 days after the operation. No complications to the surgical wound were observed. The rehabilitation had a regular course with immediate weight bearing allowed. Monthly postoperative clinical checks were scheduled for the first year. At the last examination, 1 year after the THA procedure, X-rays did not show periprosthetic fractures or implant mobilization (-Fig. 4) and the HHS was

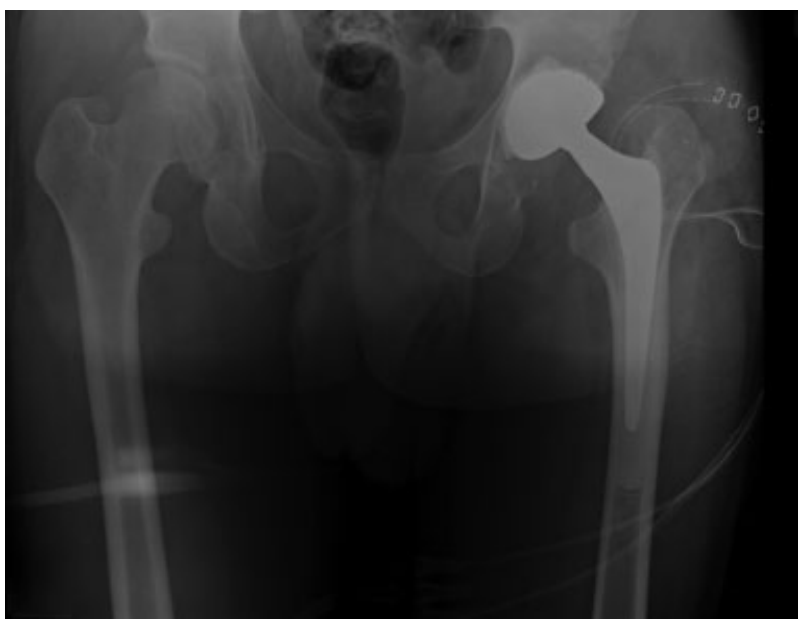

Fig. 3 Anteroposterior postoperative pelvic radiograph showing a good positioning of the prosthetic implant with no signs of fracture.

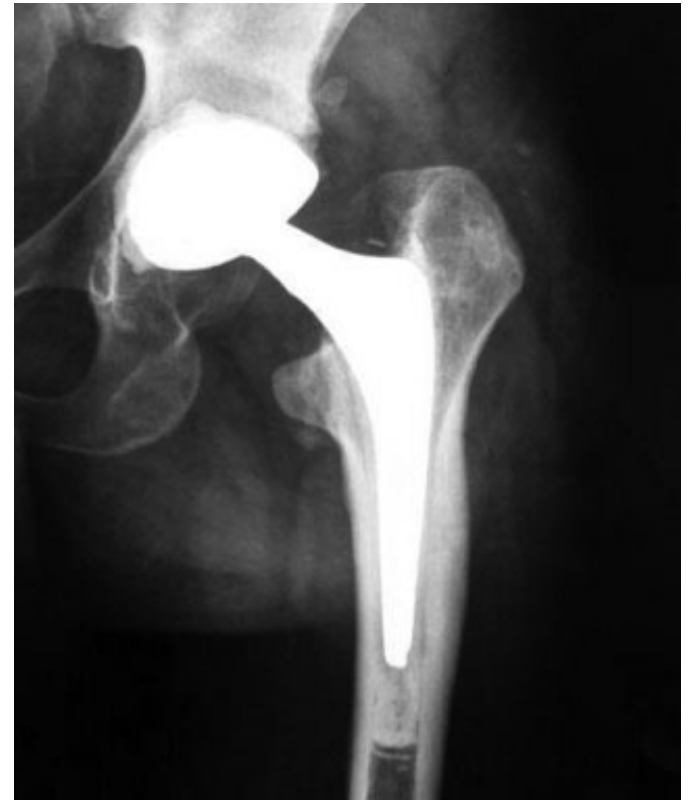

Fig. 4 Anteroposterior radiograph 1 year after surgery shows wellfixed components, no osteolysis, or mobilization of the prosthesis.

93. Improvement in HSS was due only to the "function" and the "deformity" items, pain being an irrelevant item in patients affected by CIPA.

\section{Discussion}

CIPA is a rare disorder characterized by autonomic and sensory nerves malfunction with insensitivity to both deep and superficial painful stimuli, inability to sweat and produce tears, and mild to moderate mental retardation. From an orthopaedic point of view, this condition puts the patient at risk for inveterate bone and joint fractures that could ultimately act as a predisposing factor for the development of osteomyelitis. The later a fracture is diagnosed, the bigger the damage to bone stock and surrounding tissues will be, making the surgical managing more challenging. Thus, strict and careful physical examination should be performed periodically.

Recent studies have showed that although skeletal complications are more common in the lower limbs, almost all bones and joints may be affected. Szöke in $1996^{11}$ showed that $26 \%$ of patients with CIPA presented hip disorders, and these data were confirmed by Bar-On et $\mathrm{al}^{12}$ who noted a high incidence of fractures of the lower limbs in patients with CIPA.

Fractures occurred in the early years of life due to the high activity. Another important issue related to CIPA is the risk of dislocations and infections that are not related with increasing age. ${ }^{11,12}$

In our case report, we faced an inveterate femoral neck fracture with severe head resorption that despite the young age of the patient made mandatory the implant of a hip prosthesis. To our knowledge, Erdil et al ${ }^{13}$ published the only case of a THA implanted in a patient with CIPA. The authors 
implanted a hip prosthesis in a 37-year-old Caucasian female patient using a press-fit implant due to the young age of the patient. They allowed walking with crutches at 2 days postoperatively and followed their standard antibiotic and antiembolism protocol, reporting no complications at 6 months of follow-up.

In our department, we usually implant press-fit prosthesis in young and active people, but in this case, the osteoporosis, preoperative capsular stretching, and patient's uncertainty in weight-bearing control in the postoperative period due to the absence of pain as a regulatory mechanism were the principal reasons for choosing a cemented implant with a double mobility cup. Furthermore, cemented implant allowed immediate full weight bearing, thus reducing the risk of pressure ulcers of the skin derived from lying on bed. Finally, the use of a cemented implant allowed us to reduce the risk of aseptic loosening or fracture that would have been misunderstood due to the pathology of the patient.

Limitations to our case report included the limited followup and the absence of a functional evaluation, such as computed pre- and postoperative gait analysis.

\section{References}

1 Capsoni S. From genes to pain: nerve growth factor and hereditary sensory and autonomic neuropathy type V. Eur J Neurosci 2014; 39(03):392-400

2 Dyck PJ. Neuronal atrophy and degeneration predominantly affecting peripheral sensory and autonomic neurons. In: Dick PJ, Thomas PK, Griffin JW, Low PA, Podreslo JC, eds. Peripheral Neuropathy. Philadelphia: WB Saunders; 1993:1065-1093
3 Thompson CC, Park RI, Prescott GH. Oral manifestations of the congenital insensitivity-to-pain syndrome. Oral Surg Oral Med Oral Pathol 1980;50(03):220-225

4 Rasmussen P. The congenital insensitivity-to-pain syndrome (analgesia congenita): report of a case. Int J Paediatr Dent 1996; 6(02):117-122

5 Thrush DC. Congenital insensitivity to pain. A clinical, genetic and neurophysiological study of four children from the same family. Brain 1973;96(02):369-386

6 Rosemberg S, Marie SK, Kliemann S. Congenital insensitivity to pain with anhidrosis (hereditary sensory and autonomic neuropathy type IV). Pediatr Neurol 1994;11(01):50-56

7 Brahim JS, Roberts MW, McDonald HD. Oral and maxillofacial complications associated with congenital sensory neuropathy with anhydrosis: report of two cases. J Oral Maxillofac Surg 1987; 45(04):331-334

8 Shaaban H, Bayat A, Davenport P, Shah M. Necrotising fasciitis in an infant with congenital insensitivity to pain syndrome. Br J Plast Surg 2002;55(02):160-163

9 Zhang Y, Haga N. Skeletal complications in congenital insensitivity to pain with anhidrosis: a case series of 14 patients and review of articles published in Japanese. J Orthop Sci 2014;19(05):827-831

10 Machtei A, Levy J, Friger M, Bodner L. Osteomyelitis of the mandible in a group of 33 pediatric patients with congenital insensitivity to pain with anhidrosis. Int J Pediatr Otorhinolaryngol 2011;75(04):523-526

11 Szöke G, Rényi-Vámos A, Bider MA. Osteoarticular manifestations of congenital insensitivity to pain with anhydrosis. Int Orthop 1996;20(02):107-110

12 Bar-On E, Weigl D, Parvari R, Katz K, Weitz R, Steinberg T. Congenital insensitivity to pain. Orthopaedic manifestations. J Bone Joint Surg Br 2002;84(02):252-257

13 Erdil M, Bilsel K, Imren Y, Ceylan HH, Tuncay I. Total hip arthroplasty in a patient with congenital insensitivity to pain: a case report. J Med Case Reports 2012;6:190 